\title{
Convective and wave motions in a thermal plume
}

\author{
Roman I. Kostik and Elena V. Khomenko \\ Main Astronomical Observatory, National Academy of Sciences, 03680, Kiev, Ukraine email: \\ kostik@mao.kiev.ua
}

\begin{abstract}
Here we report thermodynamical properties of a a so-called "thermal plume" observed near the solar disc center in 2001. The spectral observations of two iron lines analysed have been obtained using the Vacuum Tower Telescope (Tenerife).
\end{abstract}

We analyze wave and flow motions, thermal structure and other properties of the thermal plume which we observed in August 2001 using German Vacuum Tower Telescope (VTT) of the Observatorio del Teide (Instituto de Astrofísica de Canarias). The time series of spectral images with a total duration of about 158 minutes were acquired in two Fe I and Fe II iron lines simultaneously. The observed area was located close to the quiet solar disc center. The spectrograms were taken every $10 \mathrm{~s}$.

The observations revealed a rather stable feature which has been detected during the whole period of the observations. The size of this feature along the slit was 2000$2500 \mathrm{~km}$. The plume shows a compact brightnening in comparison with the nearby granulation. At heights around $200 \mathrm{~km}$, its intensity contrast is a factor of two larger than that of the surrounding area while reaching a factor of 4 at heights near $500 \mathrm{~km}$ (figure $1 \mathrm{a}$ ).

The following items characterize the feature during the whole period of the observations:

- The high-speed downflow of $0.5 \mathrm{~km} / \mathrm{s}$ had been seen at the continuum level. At higher photospheric layers upward flows had been observed with the modulus of the velocity increasing with height. The sign change of the velocity occurs at about $170 \mathrm{~km}$ (figure $1 b$ ).

- The velocity-intensity correlation does not exceed 0.25 at all observed heights from $0 \mathrm{~km}$ up to $500 \mathrm{~km}$ (figure 1c).

- The amplitudes of the five-minute oscillations of the velocity (figure $1 d$ ) and intensity (figure 1e) are twice lower than outside the plume.

- There is a large difference between spectral line asymmetry observed in the plume and quiet regions (figure $1 f$ ).

We conclude that the observed phenomenon has a non-convective origin. The thermodynamical properties of the atmosphere where the plume occured have been recovered from the observed profiles using the SIR inversion code. We used the spatially averaged profiles of granules, intergranular lanes and the plume doing the inversion. The plume is found to be hotter and more dense than the quiet Sun at almost all heights in the photosphere. The average magnetic field is non-zero in the plume. It can be of the order $400 \mathrm{G}$. The decrease of the amplitudes of oscillations can be attributed to the larger density and the stronger magnetic field in comparison with the surrounding atmosphere.

\section{Acknowledgements}

This work has been partially supported by the Ukrainian Foundation for Pure Researches (project 02.07/00044) and by the INTAS project 00-00084. 

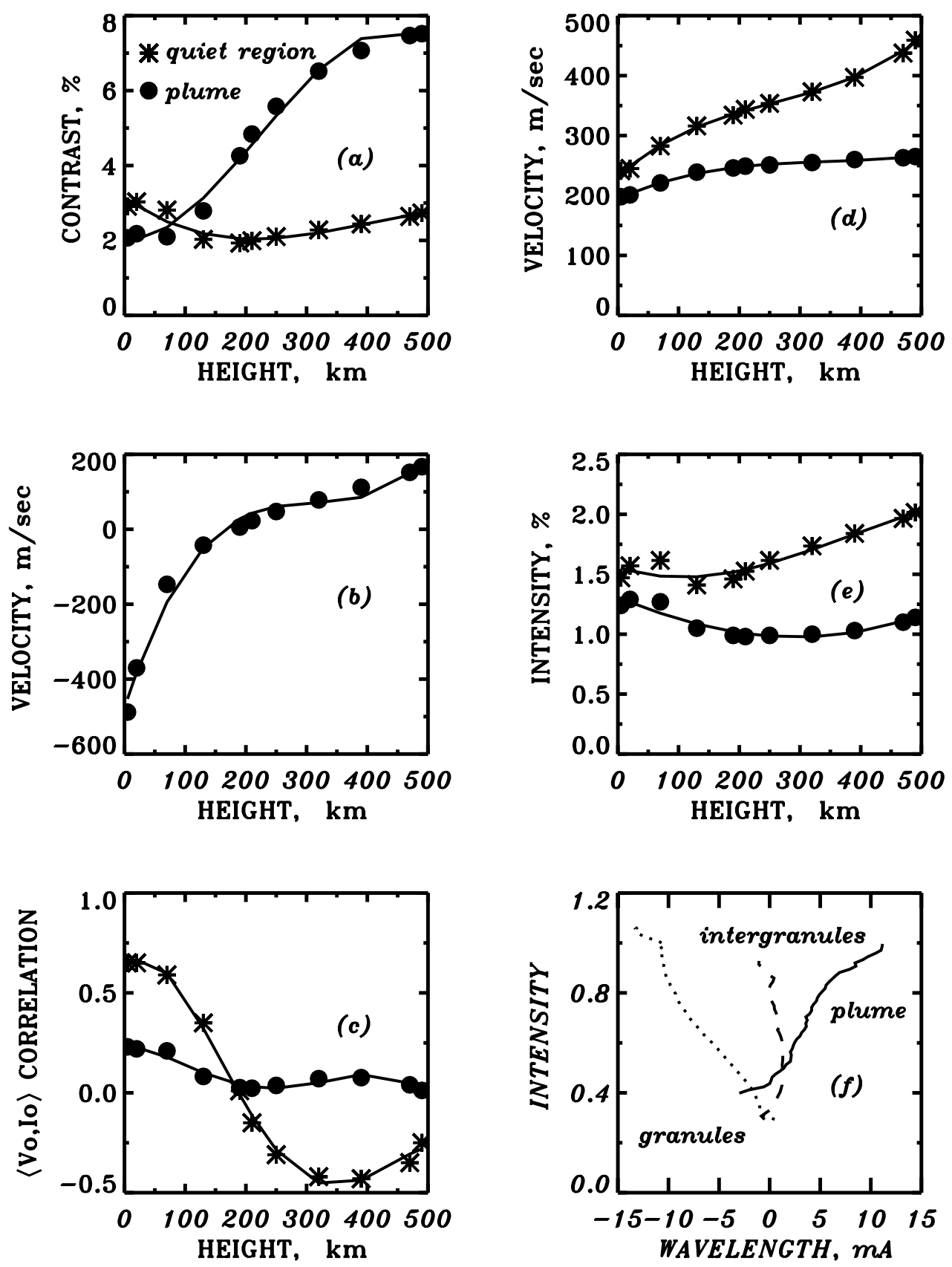

Figure 1. Height dependences in the quiet region and the plume and bisectors in granules, intergranules and in the plume. (a) Observed contrasts; $(b)$ velocity convective amplitudes; $(c)$ velocity-intensity correlations; $(d)$ velocity oscillatory amplitudes; $(e)$ intensity oscillatory amplitudes; $(f)$ temporally averaged bisectors of the Fe I line $\lambda 639.36 \mathrm{~nm}$. 